\title{
Few Differences in Metabolic Network Use Found Between Salmonella enterica Colonization of Plants and Typhoidal Mice
}

\author{
Grace Kwan ${ }^{1 \dagger}$, Brett Plagenz ${ }^{1}$, Kimberly Cowles ${ }^{1}$, Tippapha Pisithkul ${ }^{2}$, \\ Daniel Amador-Noguez ${ }^{2}$ and Jeri D. Barak ${ }^{1 *}$
}

${ }^{1}$ Department of Plant Pathology, University of Wisconsin-Madison, Madison, WI, United States, ${ }^{2}$ Department of Bacteriology, University of Wisconsin-Madison, Madison, WI, United States

\section{OPEN ACCESS}

Edited by: Nicola Holden

James Hutton Institute, United Kingdom

Reviewed by:

Shirley A. Micallef, University of Maryland, United States

Gail Preston,

University of Oxford, United Kingdom

*Correspondence:

Jeri D. Barak

jeri.barak@wisc.edu

†Present Address:

Grace Kwan,

College of Arts and Sciences, Endicott College, Beverly, MA, United States

Specialty section: This article was submitted to Plant Microbe Interactions, a section of the journal

Frontiers in Microbiology

Received: 14 December 2017 Accepted: 26 March 2018

Published: 08 May 2018

Citation:

Kwan G, Plagenz B, Cowles K, Pisithkul T, Amador-Noguez D and Barak JD (2018) Few Differences in

Metabolic Network Use Found

Between Salmonella enterica

Colonization of Plants and Typhoidal

Mice. Front. Microbiol. 9:695

doi: 10.3389/fmicb.2018.00695
The human enteric pathogen Salmonella enterica leads a cross-kingdom lifestyle, actively colonizing and persisting on plants in between animal hosts. One of the questions that arises from this dual lifestyle is how $S$. enterica is able to adapt to such divergent hosts. Metabolic pathways required for $S$. enterica animal colonization and virulence have been previously identified, but the metabolism of this bacterium on plants is poorly understood. To determine the requirements for plant colonization by S. enterica, we first screened a library of metabolic mutants, previously examined in a systemic mouse typhoidal model, for competitive plant colonization fitness on alfalfa seedlings. By comparing our results to those reported in $S$. enterica-infected murine spleens, we found that the presence of individual nutrients differed between the two host niches. Yet, similar metabolic pathways contributed to $S$. enterica colonization of both plants and animals, such as the biosynthesis of amino acids, purines, and vitamins and the catabolism of glycerol and glucose. However, utilization of at least three metabolic networks differed during the bacterium's plant- and animal-associated lifestyles. Whereas both fatty acid biosynthesis and degradation contributed to $S$. enterica animal colonization, only fatty acid biosynthesis was required during plant colonization. Though serine biosynthesis was required in both hosts, $S$. enterica used different pathways within the serine metabolic network to achieve this outcome. Lastly, the metabolic network surrounding manA played different roles during colonization of each host. In animal models of infection, O-antigen production downstream of manA facilitates immune evasion. We discovered that manA contributed to $S$. enterica attachment, to seeds and germinated seedlings, and was essential for growth in early seedling exudates, when mannose is limited. However, only seedling attachment was linked to O-antigen production, indicating that manA played additional roles critical for plant colonization that were independent of surface polysaccharide production. The integrated view of $S$. enterica metabolism throughout its life cycle presented here provides insight on how metabolic versatility and adaption of known physiological pathways for alternate functions enable a zoonotic pathogen to thrive in niches spanning across multiple kingdoms of life.

Keywords: Salmonella enterica serovar Typhimurium, plant-microbe interaction, food safety, mannose metabolism, fatty acid biosynthesis 


\section{INTRODUCTION}

The host ranges of most pathogens have boundaries that fall between the species and kingdom levels. However, a small group of microbes are able to transcend these boundaries to lead a cross-kingdom lifestyle, i.e., colonizing both animals and plants. For example, the nosocomial human pathogens Enterobacter cloacae and Serratia marcescens are also plant pathogens that cause bulb decay of onions and cucurbit yellow vine disease, respectively (Bishop and Davis, 1990; Hejazi and Falkiner, 1997; Sanders and Sanders, 1997; Bruton et al., 2003; Barak and Schroeder, 2012). Burkholderia cepacia and Pseudomonas aeruginosa are both responsible for pneumonia in cystic fibrosis patients as well as bacterial rots of onion (Barak and Schroeder, 2012). Dual pathogenicity, however, is not required for a cross-kingdom lifestyle. Xylella fastidiosa causes economically important diseases of grapes, citrus, and other plants but colonizes its insect vector without causing disease (Chatterjee et al., 2008). In order to succeed on such diverse hosts, cross-kingdom pathogens adopt strategies that range from generalist to specialist. Generalists use universal strategies to exploit animal and plant hosts, whereas specialists have dedicated mechanisms for interaction with each host (Kirzinger et al., 2011). Understanding the biology of cross-kingdom bacterial colonists on both animal and plant hosts leads to improved management of human, animal, and plant health.

This study focuses on the cross-kingdom enteric human pathogen Salmonella enterica, which colonizes agricultural plants as alternate hosts and uses them as a vector to humans (Barak and Schroeder, 2012). Numerous salmonellosis outbreaks linked to $S$. enterica-contaminated fresh produce have occurred in recent years; produce now ranks as the riskiest commodity for foodborne illness (Painter et al., 2013). Because the primary economic and pathological significance of $S$. enterica lies in the human-associated portion of its life cycle, the biology of this pathogen is much better understood in the context of its human and animal hosts compared to its plant hosts. However, in order to reduce foodborne illness, we need to understand the biology of $S$. enterica on plants, before the pathogen reaches humans and causes disease.

The cross-kingdom lifestyle of $S$. enterica requires that this bacterium attach to, colonize, and persist in divergent host environments. In animals, $S$. enterica colonizes the anaerobic intestinal lumen and causes gastroenteritis in humans (Wiedemann et al., 2014). S. enterica pathogenesis and metabolism have been best characterized in the murine host due to the tractability of mice as an experimental model. In a mouse typhoidal model, the pathogen migrates to lymphoid organs such as the spleen, invades epithelial and macrophage cells, and establishes a nutrient-limited intracellular niche called the Salmonella-containing vacuole (SCV) (Steeb et al., 2013; Wiedemann et al., 2014). Nutrients in the SCV are quantitatively limiting (i.e., low concentrations) rather than qualitatively limiting (i.e., few types of nutrients). In contrast to colonization of animal tissues, plant surfaces colonized by S. enterica are typically aerobic and sites like the rhizosphere can support high levels of bacterial replication (Kwan et al., 2015), though the nutritional drivers of this growth are still unclear. Amino acids were previously found to be limiting in alfalfa root exudates, similar to conditions encountered during colonization of the chicken intestinal lumen, but the ability of amino acid auxotrophs and transport mutants to grow in the root environment suggests that amino acid metabolism is not a determinant of $S$. enterica growth during plant association (Harvey et al., 2011; Kwan et al., 2015). A variety of sugars, organic acids, and other nutrients have also been identified on plant surfaces and in root exudates, but their contributions to plant colonization by $S$. enterica remains to be determined (El-Hamalawi and Erwin, 1986; Mercier and Lindow, 2000; Nelson, 2004; Roberts et al., 2009; Han and Micallef, 2016). The metabolic networks essential for S. enterica plant colonization are also largely unknown. Addressing this knowledge gap is critical to understanding how $S$. enterica succeeds on plants, a poorly-characterized part of the pathogen life cycle.

Differences in host physiologies and the host niches colonized by $S$. enterica led us to hypothesize that different metabolic networks are required for the distinct plant- and animalassociated lifestyles of $S$. enterica. In this study, we broadly examined the metabolic capabilities of S. enterica to identify a subset of metabolic networks that drives plant colonization by this bacterium. We used alfalfa seedlings as our model as they are the produce commodity most frequently contaminated with S. enterica (Callejón et al., 2015). By comparing the metabolic networks driving plant colonization by $S$. enterica to the known metabolic requirements for animal colonization, we provide a unique, integrated view of $S$. enterica metabolism throughout its life cycle.

\section{MATERIALS AND METHODS}

\section{Bacterial Strains and Culture Conditions}

Bacterial strains and plasmids used in this study are listed in Table 1. Strains were cultured on lysogeny broth agar (LB) medium at $37^{\circ} \mathrm{C}$ unless otherwise indicated. When necessary, antibiotics were added at the following concentrations: ampicillin (Amp), $100 \mu \mathrm{g} / \mathrm{ml}$; chloramphenicol (Cm), $40 \mu \mathrm{g} / \mathrm{ml}$; kanamycin (Kan), $50 \mu \mathrm{g} / \mathrm{ml}$.

\section{Mutant Construction}

Gene deletion mutants were generated in S. enterica KHLT2 with the $\lambda$-Red recombination method (Datsenko and Wanner, 2000) and transduced into S. enterica 14028 S or S. enterica SL1344 by bacteriophage P22. A S. enterica 14028S $\Delta r f b P::$ Kan mutant was obtained from a deletion library generated by the same method (Santiviago et al., 2009). Due to lack of O-antigen production, this mutant could not be transduced into the SL1344 background. All mutants were confirmed by PCR, either with two flanking verification ("veri") primers or one flanking verification primer and an antibiotic-cassette specific primer (c1 or k2) (Datsenko and Wanner, 2000). Mutagenesis and verification primers are listed in Table 2. Double mutants were produced by transduction into a mutant background. 
TABLE 1 | List of strains.

\begin{tabular}{|c|c|c|}
\hline $\begin{array}{l}\text { Strain or } \\
\text { plasmid }\end{array}$ & Genotype/Relevant markers & References \\
\hline \multicolumn{3}{|l|}{ STRAINS } \\
\hline \multicolumn{3}{|c|}{ S. enterica sv. Typhimurium, SL1344 } \\
\hline & WT; hisG rpsL, St ${ }^{R}$ & Hoiseth and Stocker, 1981 \\
\hline & $\Delta f a d D:: \operatorname{Kan}^{R} \Delta f a d K:: \mathrm{Cm}^{\mathrm{R}}, \mathrm{St}^{\mathrm{R}}$ & Steeb et al., 2013 \\
\hline & $\begin{array}{l}\Delta g / p F K:: \operatorname{Kan}^{\mathrm{R}} \Delta g / d A:: \mathrm{Cm}^{\mathrm{R}} \Delta g / p T \\
\Delta u g p B, \mathrm{St}^{\mathrm{R}}\end{array}$ & Steeb et al., 2013 \\
\hline & $\Delta r b s B:: \mathrm{Cm}^{\mathrm{R}}, \mathrm{St}^{\mathrm{R}}$ & Steeb et al., 2013 \\
\hline & $\Delta g a l P:: \operatorname{Kan}^{R} \Delta m g l B:: \mathrm{Cm}^{\mathrm{R}}, \mathrm{St}^{\mathrm{R}}$ & Steeb et al., 2013 \\
\hline & $\Delta$ nanT $:: \mathrm{Cm}^{\mathrm{R}}, \mathrm{St}^{\mathrm{R}}$ & Steeb et al., 2013 \\
\hline & $\Delta \operatorname{man} X:: \operatorname{Kan}^{\mathrm{R}}, \mathrm{St} \mathrm{R}^{\mathrm{R}}$ & Steeb et al., 2013 \\
\hline & $\Delta h i s P:: \mathrm{Kan}^{\mathrm{R}} \Delta \operatorname{artP}:: \mathrm{Cm}^{\mathrm{R}}, \mathrm{St}^{\mathrm{R}}$ & Steeb et al., 2013 \\
\hline & $\Delta m a / G:: \mathrm{Cm}^{R}, \mathrm{St}^{\mathrm{R}}$ & Steeb et al., 2013 \\
\hline & $\Delta \operatorname{man} X:: \operatorname{Kan}^{R} \Delta n a g E:: \mathrm{Cm}^{\mathrm{R}}, \mathrm{St}^{\mathrm{R}}$ & Steeb et al., 2013 \\
\hline & $\Delta d e o P:: \mathrm{Cm}^{\mathrm{R}}, \mathrm{St}^{\mathrm{R}}$ & Steeb et al., 2013 \\
\hline & $\Delta / / P:: \operatorname{Kan}^{\mathrm{R}}, \mathrm{St}^{\mathrm{R}}$ & Steeb et al., 2013 \\
\hline & $\Delta f u c P:: K_{a n}^{R}, S^{R}$ & Steeb et al., 2013 \\
\hline & $\Delta$ eutC:: $\operatorname{Kan}^{\mathrm{R}}, \mathrm{St}^{\mathrm{R}}$ & Steeb et al., 2013 \\
\hline & $\Delta$ proP:: $\operatorname{Kan}^{\mathrm{R}} \Delta$ proW:: $\mathrm{Cm}^{\mathrm{R}}, \mathrm{St}^{\mathrm{R}}$ & Steeb et al., 2013 \\
\hline & $\begin{array}{l}\Delta p t s G:: \operatorname{Kan}^{R} \Delta \operatorname{man} X \Delta m g l B \Delta g a l P, \\
S t^{R}\end{array}$ & Steeb et al., 2013 \\
\hline & $\Delta$ exuT::Kan ${ }^{R}, \mathrm{St}^{\mathrm{R}}$ & Steeb et al., 2013 \\
\hline & $\Delta S T M 4432:: \operatorname{Kan}^{\mathrm{R}}, \mathrm{St}^{\mathrm{R}}$ & Steeb et al., 2013 \\
\hline & $\Delta i d n T:: \operatorname{Kan}^{\mathrm{R}} \Delta g n t T:: \mathrm{Cm}^{\mathrm{R}}, \mathrm{St}^{\mathrm{R}}$ & Steeb et al., 2013 \\
\hline & $\Delta / y s P:: \operatorname{Kan}^{R} \Delta h i s P, \mathrm{St}^{\mathrm{R}}$ & Steeb et al., 2013 \\
\hline & suhpT::Kan ${ }^{R}, \mathrm{St}^{\mathrm{R}}$ & Steeb et al., 2013 \\
\hline & 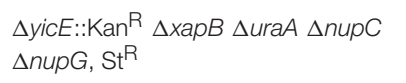 & Steeb et al., 2013 \\
\hline & $\Delta g l g P \Delta$ malP $, \mathrm{St}^{\mathrm{R}}, \mathrm{Cm}^{\mathrm{R}}, \mathrm{Kan}^{\mathrm{R}}$ & Steeb et al., 2013 \\
\hline & $\Delta m t / A \Delta m t / D, \mathrm{St}^{\mathrm{R}}, \mathrm{Cm}^{\mathrm{R}}, \mathrm{Kan}^{\mathrm{R}}$ & Steeb et al., 2013 \\
\hline & $\Delta g / p F K:: K_{a n}{ }^{R} \Delta g / p T \Delta u g p B, S_{t}^{R}$ & Steeb et al., 2013 \\
\hline & $\Delta g / p F K:: \mathrm{Kan}^{\mathrm{R}}, \mathrm{St}^{\mathrm{R}}$ & Steeb et al., 2013 \\
\hline & $\Delta l y s A:: \operatorname{Kan}^{R} \Delta c a d A, \mathrm{St}^{\mathrm{R}}$ & Steeb et al., 2013 \\
\hline & $\begin{array}{l}\Delta \text { sifB::GFPweak::Kan }{ }^{R} \Delta y a b J \Delta \text { thil, } \\
\text { St }^{R}\end{array}$ & Steeb et al., 2013 \\
\hline & $\begin{array}{l}\Delta \operatorname{man} X:: \mathrm{Kan}^{\mathrm{R}} \Delta m a n A:: \mathrm{Cm}^{\mathrm{R}} \\
\text { sifB@GFPs, St }\end{array}$ & Steeb et al., 2013 \\
\hline & $\Delta m a n A:: \mathrm{Cm}^{\mathrm{R}}$ sifB@GFPs, $\mathrm{St}^{\mathrm{R}}$ & Steeb et al., 2013 \\
\hline & $\Delta p d x A \Delta S T M 0163$ sifB@sYFP, St ${ }^{R}$ & Steeb et al., 2013 \\
\hline & $\Delta l y s A:: \operatorname{Kan}^{R}, S t^{R}$ & Steeb et al., 2013 \\
\hline & $\Delta i / v D:: \operatorname{Kan}^{\mathrm{R}}, \mathrm{St}^{\mathrm{R}}$ & Steeb et al., 2013 \\
\hline & $\Delta$ thrC:: $\mathrm{Kan}^{\mathrm{R}}, \mathrm{St}^{\mathrm{R}}$ & Steeb et al., 2013 \\
\hline & $\begin{array}{l}\Delta \operatorname{trp} A:: \mathrm{Kan}^{\mathrm{R}} \Delta p h e A \Delta t y r A, \mathrm{St}^{\mathrm{R}} \\
\Delta p \operatorname{proC}:: \mathrm{Cm}^{\mathrm{R}}, \mathrm{St}^{\mathrm{R}}\end{array}$ & $\begin{array}{l}\text { Steeb et al., } 2013 \\
\text { Steeb et al., } 2013\end{array}$ \\
\hline & $\Delta c y s E:: \mathrm{Kan}^{\mathrm{R}} \Delta m e t A:: \mathrm{Cm}^{\mathrm{R}} \mathrm{St}^{\mathrm{R}}$ & Steeb et al., 2013 \\
\hline & $\Delta l e u B:: \mathrm{Cm}^{\mathrm{R}}, \mathrm{St}^{\mathrm{R}}$ & Steeb et al., 2013 \\
\hline & $\Delta$ purH:: $\mathrm{Cm}^{\mathrm{R}}, \mathrm{St}^{\mathrm{R}}$ & Steeb et al., 2013 \\
\hline & $\Delta$ purA::Kan ${ }^{R}, S^{R}$ & Steeb et al., 2013 \\
\hline & $\Delta t s x:: \mathrm{Cm}^{\mathrm{R}}, \mathrm{St}^{\mathrm{R}}$ & Steeb et al., 2013 \\
\hline JDB 1332 & $\begin{array}{l}\Delta p d x A \Delta S T M 0163 \text { sifB@sYFP } \\
+ \text { pKTKan, } S^{R}\end{array}$ & This study \\
\hline JDB 1339 & $\Delta f a b G:: C m R, S^{R}$ & This study \\
\hline JDB 1343 & $\Delta f a b G:: C m R+p E V S 141\left(\operatorname{Kan}^{R}\right), \mathrm{St}^{R}$ & This study \\
\hline
\end{tabular}

(Continued)
TABLE 1 | Continued

\begin{tabular}{|c|c|c|}
\hline $\begin{array}{l}\text { Strain or } \\
\text { plasmid }\end{array}$ & Genotype/Relevant markers & References \\
\hline JDB 1344 & $\begin{array}{l}\Delta f a b G:: C m R+ \\
\text { pEVS141 }+ \text { gfp::fabHDG(KanR), StR }\end{array}$ & This study \\
\hline JDB 1336 & $\Delta g m d:: \operatorname{Kan}^{\mathrm{R}}, \mathrm{St}^{\mathrm{R}}$ & This study \\
\hline JDB 1337 & $\Delta m a n A:: \mathrm{Cm}^{\mathrm{R}}, \mathrm{St}^{\mathrm{R}}$ & This study \\
\hline JDB 1340 & 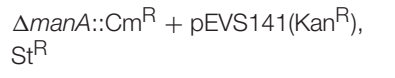 & This study \\
\hline JDB 1341 & 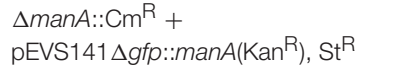 & This study \\
\hline JDB 1399 & $\Delta \operatorname{ser} A:: \mathrm{Cm}^{\mathrm{R}}, \mathrm{St}^{\mathrm{R}}$ & This study \\
\hline JDB 1400 & $\begin{array}{l}\Delta s e r A:: \mathrm{Cm}^{R}+ \\
\text { pEVS141 } \Delta g f p:: \operatorname{ser} A\left(\operatorname{Kan}^{R}\right), \mathrm{St}^{R}\end{array}$ & This study \\
\hline JDB 1342 & $W T+p E V S 141\left(\operatorname{Kan}^{R}\right)$ & This study \\
\hline \multicolumn{3}{|c|}{ S. enterica sv. Typhimurium, $14028 \mathrm{~S}$} \\
\hline & WT & ATCC \\
\hline & $\Delta a s n A:: K a n \quad \Delta a s n B:: C m$ & Kwan et al., 2015 \\
\hline & $\Delta g l y A:: \operatorname{Tn} 10 \mathrm{~d}$ & Kwan et al., 2015 \\
\hline & $\Delta / y s A:: K a n$ & Kwan et al., 2015 \\
\hline & $\Delta m e t C::$ Kan & Kwan et al., 2015 \\
\hline & $\Delta p h e A::$ Kan & Kwan et al., 2015 \\
\hline & proC693::MudA & Kwan et al., 2015 \\
\hline & $\Delta \operatorname{trpB}::$ Kan & Hao et al., 2012 \\
\hline & $\Delta t y r A:: K a n$ & Kwan et al., 2015 \\
\hline & $\Delta r f b P:: K_{a n}^{R}$ & Santiviago et al., 2009 \\
\hline JDB 1358 & $\Delta g m d:: \operatorname{Kan}^{\mathrm{R}}$ & This study \\
\hline \multicolumn{3}{|c|}{ PLASMIDS } \\
\hline pKTKan & $g f p, \mathrm{Kan}^{\mathrm{R}}$ & Miller et al., 2000 \\
\hline pEVS141 & $g f p, \mathrm{Kan}^{\mathrm{R}}$ & Dunn et al., 2006 \\
\hline
\end{tabular}

\section{Complementation}

The $\Delta m a n A:: \mathrm{Cm}, \Delta f a b G:: \mathrm{Cm}$, and $\Delta s e r A:: \mathrm{Cm}$ strains were genetically complemented by cloning of the $\operatorname{manA}$, fabHDG, or serA genes into pEVS141. The complementation plasmids were transformed into the respective mutant strains. Transformants were selected on LB+Kan and confirmed by PCR of the cloned gene(s) from extracted plasmid DNA. The same primers were used for cloning and PCR confirmation (Table 2). For chemical complementation, $0.2 \mathrm{mM}$ mannose was provided to $\triangle$ manA:: $\mathrm{Cm} ; 0.1 \mathrm{mM}$ cysteine was added as a supplement for $\Delta c y s E \Delta m e t A$.

\section{Preparation of Inoculum}

For all experiments except mutant analysis of serA, overnight bacterial cultures were suspended in sterile deionized water and normalized to $\sim 10^{8} \mathrm{CFU} / \mathrm{ml}$ by absorbance at $\mathrm{OD}_{600}$ using a spectrophotometer. This suspension was diluted as needed. For experiments involving $\Delta \operatorname{ser} A:: \mathrm{Cm}$, the WT, mutant, and complement bacterial suspensions were made from overnight cultures grow in liquid lysogeny broth rather than LB agar. Inoculum populations were verified by dilution plating on LB. 
TABLE 2 | Primers used in this study.

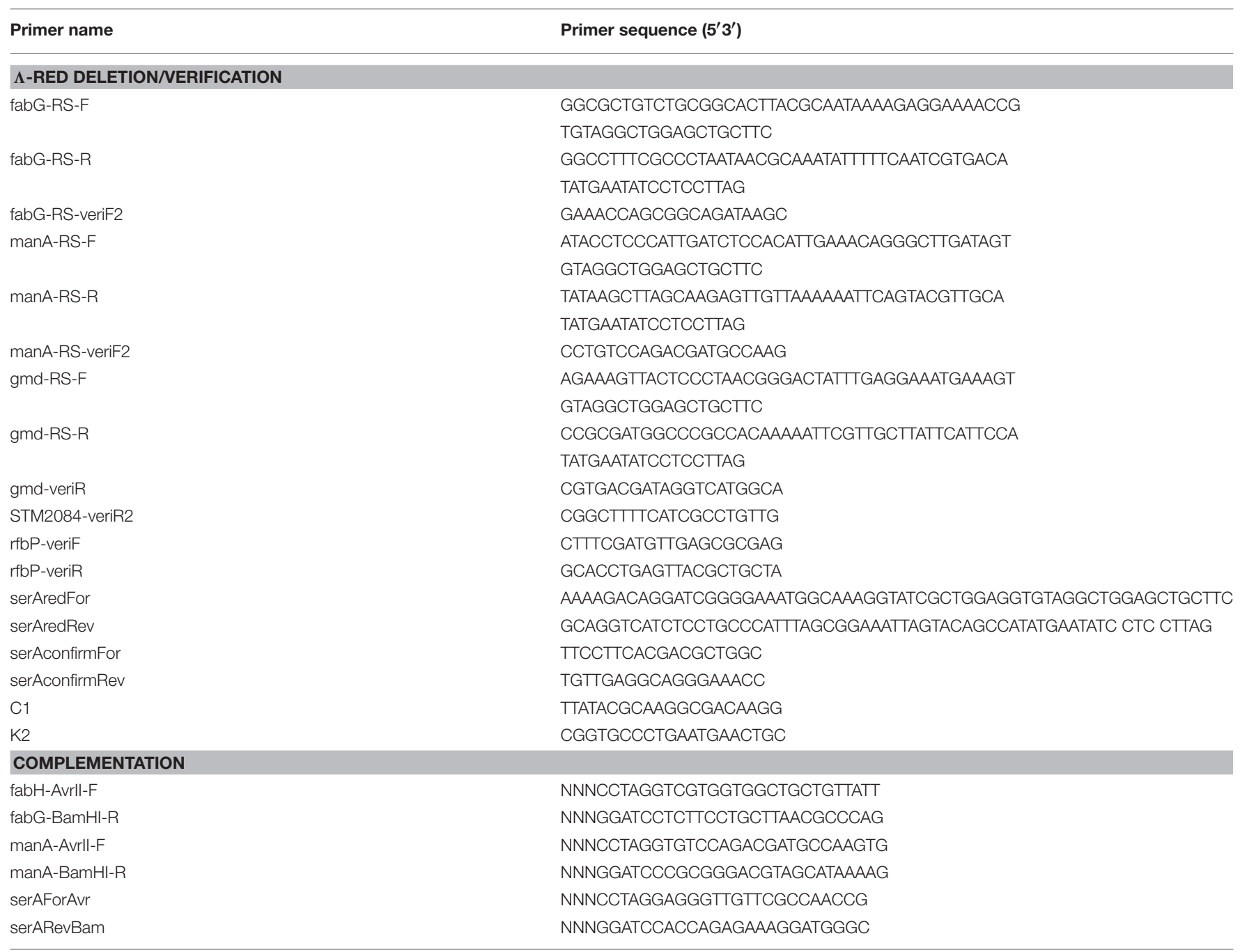

\section{Plant Assays}

Surface sanitized alfalfa seeds (International Specialty Supply) were prepared as described by Cowles et al. (2016).

For alfalfa attachment assays, 10 seeds or 10 3-day-old seedlings were placed in sterile $50 \mathrm{ml}$ conical tubes and coinoculated with $20 \mathrm{ml}$ of a $10^{3} \mathrm{CFU} / \mathrm{ml}$ bacterial suspension of wild-type (WT) and mutant at a 1:1 $( \pm 0.1)$ ratio. For experiments using $\triangle \operatorname{man} A:: \mathrm{Cm}$, the WT, mutant, and complement bacterial suspensions were made from overnight cultures on M9 minimal agar media amended with $0.4 \%$ glucose and $0.2 \mathrm{mM}$ histidine because LB likely contains low concentrations of mannose (our own unpublished results). Histidine was required because S. enterica SL1344 is a histidine auxotroph. For chemical complementation, mannose was added to this overnight culture because the surface polymers produced downstream of manA (see Discussion) that are known to mediate attachment must be pre-formed in culture; $S$. enterica does not grow during the first $4 \mathrm{~h}$ following inoculation into germinating seedling culturesthe time period used for this attachment assay (Becker et al.,
2006; Kwan et al., 2015). Tubes were incubated for $4 \mathrm{~h}$ at room temperature with gentle shaking. Then, the water was decanted and seeds or seedlings were rinsed twice in $20 \mathrm{ml}$ sterile water by vortexing on high for $30 \mathrm{~s}$ to remove loosely attached bacterial cells. Individual seeds or seedlings were homogenized in 300 ul sterile water and plated on $\mathrm{LB}$ and $\mathrm{LB}+\mathrm{Kan}$ or $\mathrm{LB}+\mathrm{Cm}$. Plates were incubated at $37^{\circ} \mathrm{C}$ overnight, and populations were enumerated. The experiment was repeated at least three times.

For alfalfa colonization assays, $0.3 \mathrm{~g}$ surface sanitized alfalfa seeds were placed in plastic petri dishes and irrigated with $20 \mathrm{ml}$ of a bacterial suspension containing $10^{5} \mathrm{CFU} / \mathrm{ml}$. For co-inoculation (competition) assays, the bacterial suspension contained a 1:1 $( \pm 0.1)$ ratio of two bacterial strains. After a $1 \mathrm{~h}$ incubation at room temperature with constant gentle agitation, the irrigation water was replaced with fresh sterile water. Inoculated seeds were then incubated at $24^{\circ} \mathrm{C}$ on a rotating shaker set at $\sim 40 \mathrm{rpm}$ and under constant light. Every $24 \mathrm{~h}$, samples were removed, and the irrigation water was replaced. Seedlings with attached seed coats were sampled at $24 \mathrm{~h}$ 
post-inoculation, and those without seed coats were selected at 48 and $72 \mathrm{~h}$. At each time point, three seedlings were placed in a $50 \mathrm{ml}$ conical tube containing $20 \mathrm{ml}$ sterile water and vortexed on high for $30 \mathrm{~s}$. Then, individual seedlings were homogenized in $500 \mu \mathrm{l}$ sterile water and dilution plated on LB, LB+Kan, and/or $\mathrm{LB}+\mathrm{Cm}$. Plates were incubated at $37^{\circ} \mathrm{C}$ overnight, and then populations were enumerated. There were three replicates per treatment and the experiment was repeated at least three times. Because it was necessary to distinguish between WT and mutant cells in our competition assays (detailed below), pKT-Kan was transformed into the unmarked $\Delta p d x A \Delta$ STM0163 strain as a selection marker. No growth difference was observed between the marked and unmarked strains in LB (data not shown). For chemical complementation, the appropriate metabolite was added into the irrigation water at each water change.

For growth assays in the exudates of germinating plant seedlings, experiments were set up as described for the colonization assays above except that the inoculum concentration was $10^{3} \mathrm{CFU} / \mathrm{ml}$ and the irrigation water was never changed. Growth was examined in the exudates of three different plants: alfalfa, broccoli, and lettuce. Broccoli seeds (Johnny Seeds; Winslow, ME) were surface sanitized as described above for alfalfa seeds. Lettuce seeds (cultivar Green Towers; GeneFresh Technologies; Salinas, CA) were not sanitized because bleach sanitized seeds failed to germinate. Approximately 130 alfalfa $(=0.3 \mathrm{~g})$, lettuce $(=0.125 \mathrm{~g})$, or broccoli seeds (counted by hand) were placed in each petri plate. Planktonic bacterial cell populations were determined every $24 \mathrm{~h}$, for $72 \mathrm{~h}$, by removing $100 \mathrm{ul}$ of the irrigation water and plating serial dilutions on LB. Plates were incubated at $37^{\circ} \mathrm{C}$ overnight and then populations were enumerated. No bacterial growth was observed for control samples that were not inoculated (data not shown). There were three replicates per treatment, and the experiment was repeated at least three times.

To determine $\Delta$ manA::Cm growth in more mature plant exudates, the experiment was conducted identically to the typical growth assay (above) except that alfalfa seeds were germinated in sterile water for $24 \mathrm{~h}$ prior to bacterial inoculation. This method contrasts with the normal experimental setup where bacteria were inoculated into sterile water containing yet ungerminated seeds.

\section{Seedling Toxicity Assays}

Alfalfa seeds $(0.3 \mathrm{~g})$ were germinated in $20 \mathrm{ml}$ of a $0.1 \mathrm{mM}$ cysteine solution and incubated for 3 days at $24^{\circ} \mathrm{C}$ on a rotating shaking set at $\sim 40 \mathrm{rpm}$ and under constant light. Seeds germinated in sterile water served as the control. The irrigation water was removed and replaced with sterile water or $0.1 \mathrm{mM}$ cysteine daily. The length of five 3-day-old seedlings was determined using a ruler. The experiment was repeated three times.

\section{Metabolomics}

Metabolites in alfalfa seedling exudates were identified by liquid chromatography-mass spectrometry (LC-MS) as described by Kwan et al. (2015). Briefly, $0.3 \mathrm{~g}$ surface sanitized alfalfa seeds were germinated in $20 \mathrm{ml}$ of a S. enterica $14028 \mathrm{~S}$ bacterial suspension $\left(10^{3} \mathrm{CFU} / \mathrm{ml}\right)$ or mock-inoculated with sterile water. At $0,8,24,48$, and $72 \mathrm{~h}$, the irrigation water was removed, passed through a $0.22 \mu \mathrm{M}$ PES membrane filter, and analyzed by LCMS to identify metabolites present in alfalfa seedling exudates. At least two biological replicates, each produced by pooling three technical replicates collected concurrently, were analyzed for each treatment at each time point.

\section{Statistical Analyses}

Statistical analyses were performed using $\mathrm{R}$ software (version 2.14.1; R Development Core Team, R Foundation for Statistical Computing, Vienna, Austria [http://www.R-project.org]). Bacterial population counts were $\log _{10}$-transformed prior to analysis except in seed and seedling attachment assays where counts remained untransformed. The mean population size (single inoculation) or percent total population (co-inoculation) were calculated at each time point for each experiment and then averaged across experiments. Biological replicates within an experiment were considered subsamples. For plant attachment assays, the percent of the total population comprised of the mutant at each time point was compared against the percentage of the mutant in the inoculum in paired, two-tailed $t$-tests assuming unequal variance. Differences among treatments at each time point in singly inoculated growth (alfalfa) and seedling toxicity were also evaluated by paired, two-tailed $t$-tests assuming unequal variance. Differences in growth between amino acid auxotrophs and WT in lettuce and broccoli seedling exudates were calculated. These differences were rank transformed (Conover, 2012), then compared to an expected value $\mu, 0$, using one-sample $t$-tests. For plant co-colonization and co-growth assays, the average percent total population for each mutant was compared against the expected value of $\mu, 50$, in one-sample $t$-tests. For plant complementation assays, differences among treatments at each time point were analyzed using Tukey's honestly significant difference (HSD) test (based on two-way analysis of variance [ANOVA] with strain and experiment as factors). Significance was set at a $p<0.05$ for all aforementioned tests.

The ratios of nutrient concentrations in the presence and absence of $S$. enterica were compared against an expected $\mu$ value of 1 using one-sample $t$-tests $(p<0.1)$. A ratio of 1 indicates that $S$. enterica had no effect on the concentration of a particular nutrient in alfalfa exudates; a ratio less than 1 indicates nutrient depletion in the presence of S. enterica.

\section{RESULTS}

\section{Metabolic Network Requirements for S. enterica Plant Colonization}

To identify metabolic networks that contribute to plant colonization by $S$. enterica, we screened a library of $S$. enterica SL1344 mutants for colonization fitness on alfalfa seedlings (Figure 1). This set of mutants had previously been tested for spleen colonization in a mouse typhoid fever model (Steeb et al., 2013). Mutants defective in the biosynthesis of amino acids, vitamins, and purines were outcompeted by the isogenic WT, suggesting that these nutrients are limiting in or on seedling 


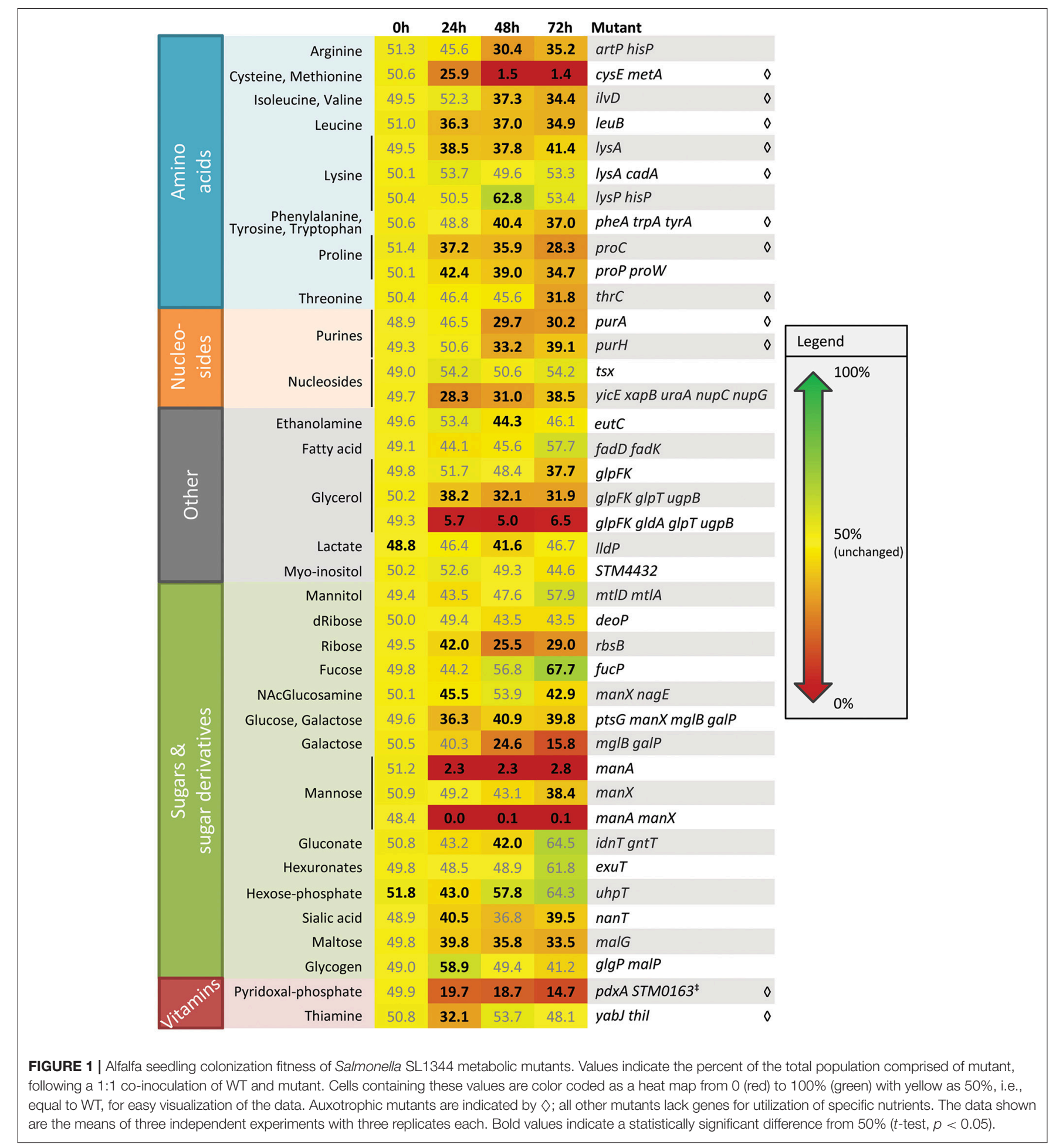

surfaces. Amino acid auxotrophs were also reduced in growth in alfalfa (Kwan et al., 2015), broccoli, and lettuce seedling exudates, indicating that amino acid limitation is common in the plant environment (Table S1). A cysE metA mutant, unable to biosynthesize the sulfur-containing amino acids cysteine and methionine, was severely compromised in its competitive colonization fitness vs. the WT, especially at 48 and $72 \mathrm{~h}$.
This fitness defect was partially complemented by addition of $0.1 \mathrm{mM}$ cysteine but alfalfa seedlings were severely stunted (Figure S1). Seedlings germinated in the presence of cysteine were $\sim 50 \%$ shorter than their counterparts irrigated with water only, indicating that this amino acid is toxic to alfalfa development. Biosynthesis of many compounds is required for $S$. enterica colonization of both plant and animal hosts. 
In addition to biosynthesis, nutrient transport also contributes to $S$. enterica plant colonization (Figure 1). A glpFK gldA $g l p T$ $u g p B$ mutant, unable to transport or utilize glycerol and glycerol3-phosphate, had a severe competitive disadvantage vs. the WT, suggesting that glycerol and glycerol-3-phosphate are major nutrient sources that drive $S$. enterica seedling colonization. Based on the mutant data, glucose, galactose, ribose, maltose, sialic acid, nucleosides, arginine, and proline are also likely present in the seedling environment and used to support bacterial colonization of seedlings. The temporal releases of nutrients in alfalfa exudates that can serve as sole carbon or nitrogen sources for S. enterica, as inferred from the mutant data and as directly measured by LC-MS (Tables S2-S4) are detailed in Table 3. Utilization of a variety of plant-derived nutrients, likely simultaneously, contributes to bacterial fitness during plant colonization.

\section{Fatty Acid Biosynthesis, but Not Catabolism, Is Required for Plant Colonization by S. enterica}

Proteomic data indicate that the $S$. enterica fatty acid metabolic network is active during mouse spleen colonization and alfalfa seedling-associated growth (Figure 2A; Steeb et al., 2013; Kwan et al., 2015). Both degradation and biosynthesis of fatty acids are active in the animal model of infection and mutants defective in these pathways are less fit (Becker et al., 2006; Steeb et al., 2013). In contrast, fatty acid degradation appears to be inactive or dispensable for plant colonization. No proteins involved in $\beta$ oxidation of fatty acids were detected in a shotgun proteomic survey of the $S$. enterica proteome during growth in alfalfa seedling exudates (Kwan et al., 2015), and a fadD fadK mutant, unable to transport or activate fatty acids for $\beta$-oxidation, had no loss of fitness during competitive colonization of alfalfa seedlings (Figure 1). However, five proteins involved in fatty acid biosynthesis (AccA, AccB, AccC, AccD, and FabB) were identified in the $S$. enterica proteome during seedling-associated growth (Kwan et al., 2015), suggesting that this metabolic pathway was both active and required by $S$. enterica in this environment. To test this hypothesis, we examined a $f a b G$ mutant, defective in fatty acid biosynthesis, and found that it was non-competitive against the WT for seedling colonization (Figure 2B). The fabG mutant never exceeded $0.06 \%$ of the total bacterial population and frequently no $f a b G$ colonies were recovered. This mutant also exhibited a severe colonization defect even in the absence of competition from WT (Figure 2C). To better understand the nature of the defect, we examined the $f a b G$ mutant in each of the two distinct phases of plant colonization, attachment to plant surfaces followed by microbial growth. The fab $G$ mutant was as competitive as WT for attachment to alfalfa seeds and 3-day-old seedlings (data not shown) but showed a growth defect in germinating alfalfa seedling exudates (Figure 2D). Both the growth and colonization defects of the fabG mutant were complemented by a plasmid-borne WT copy of fabG (Figures 2C,D). Taken together, fatty acid biosynthesis is not just a fitness factor but a determinant of growth during early seedling colonization by $S$. enterica.
TABLE 3 | Temporal utilization of nutrients by S. enterica in germinating alfalfa exudates that serve as sole $\mathrm{C}$ - or $\mathrm{N}$-sources ${ }^{\star}$ or vitamins.

\begin{tabular}{|c|c|c|c|c|c|c|}
\hline Nutrient type & Nutrient & & & $24 \mathrm{~h}$ & $48 \mathrm{~h}$ & $72 \mathrm{~h}$ \\
\hline \multirow[t]{8}{*}{ Amino acids } & Aspartate & $\ddagger$ & & $x$ & $x$ & $x$ \\
\hline & Glutamine & $\ddagger$ & $\S$ & $X$ & $X$ & $x$ \\
\hline & Glycine & & $\S$ & $x$ & $x$ & $x$ \\
\hline & Proline & $\ddagger$ & $\S$ & $x$ & $x$ & $x$ \\
\hline & Serine & $\ddagger$ & $\S$ & $X$ & $X$ & $x$ \\
\hline & Alanine & $\ddagger$ & & - & $X$ & $x$ \\
\hline & Arginine & $\ddagger$ & $\S$ & - & $x$ & $x$ \\
\hline & Asparagine & $\ddagger$ & & - & X & $x$ \\
\hline \multirow[t]{4}{*}{ Nucleosides } & Adenosine & $\ddagger$ & & - & $X$ & $x$ \\
\hline & Guanosine & $\ddagger$ & & - & $x$ & $x$ \\
\hline & Uridine & $\ddagger$ & & - & $X$ & $x$ \\
\hline & Inosine & $\ddagger$ & & - & $X$ & $X$ \\
\hline \multirow[t]{4}{*}{ Organic acids } & Fumarate & $\ddagger$ & & - & $x$ & $x$ \\
\hline & Glycerate & $\ddagger$ & & - & $x$ & $x$ \\
\hline & Malate & ‡ & & - & $X$ & $X$ \\
\hline & Succinate & $\ddagger$ & & - & $X$ & $x$ \\
\hline \multirow[t]{2}{*}{ Polyols } & Glycerol-3-P & & $\S$ & $x$ & $X$ & $X$ \\
\hline & Glycerol & & $\S$ & - & - & $x$ \\
\hline \multirow[t]{4}{*}{ Sugars } & Maltose & & $\S$ & $x$ & $x$ & $x$ \\
\hline & Ribose & & $\S$ & $x$ & $X$ & $x$ \\
\hline & Glucose & & $\S$ & $x$ & - & - \\
\hline & Galactose & & $\S$ & - & $x$ & $x$ \\
\hline \multirow[t]{4}{*}{ Vitamins } & Biotin & $\ddagger$ & & - & $X$ & $x$ \\
\hline & Panthothenate & $\ddagger$ & & - & $X$ & $x$ \\
\hline & Pyridoxal-P & & $\S$ & $x$ & $X$ & $x$ \\
\hline & Thiamine & $\ddagger$ & $\S$ & $x$ & $x$ & $x$ \\
\hline
\end{tabular}

"Compounds that can be used as sole carbon and nitrogen sources by S. enterica were identified by Gutnick et al. (1969). ‡, Nutrient utilization directly measured by LCMS, as reported in Kwan et al. (2015) and this work. \$, Nutrient utilization inferred from mutant data. Glutamine utilization inferred from Kwan et al. (2015). X, present and utilized by S. enterica as a nutrient. -, not present or no significant utilization by S. enterica as a nutrient. All nutrients measured by LC-MS were detected in plant exudates at all time points indicated by "-.." Inferences made from mutant phenotypes were unable to distinguish between nutrient absence vs. lack of utilization by S. enterica.

\section{Serine Biosynthesis Is Required for Plant Colonization by S. enterica}

During alfalfa seedling-associated S. enterica growth and animal infection, serine metabolic network activity was detected (Figure 3A; Steeb et al., 2013; Kwan et al., 2015) and yet, serine biosynthesis is dispensable for mouse infection (Jelsbak et al., 2014). A serA mutant was poorly competitive for alfalfa seedling colonization at 48 and $72 \mathrm{hpi}$ (Figure 3B) and grew poorly in root exudates (data not shown). The fitness of the serA mutant was fully restored by genetic complementation by a plasmid carrying a WT copy of serA. These data suggest that serine biosynthesis occurs during both plant and animal colonization but is only essential during seedling colonization. 

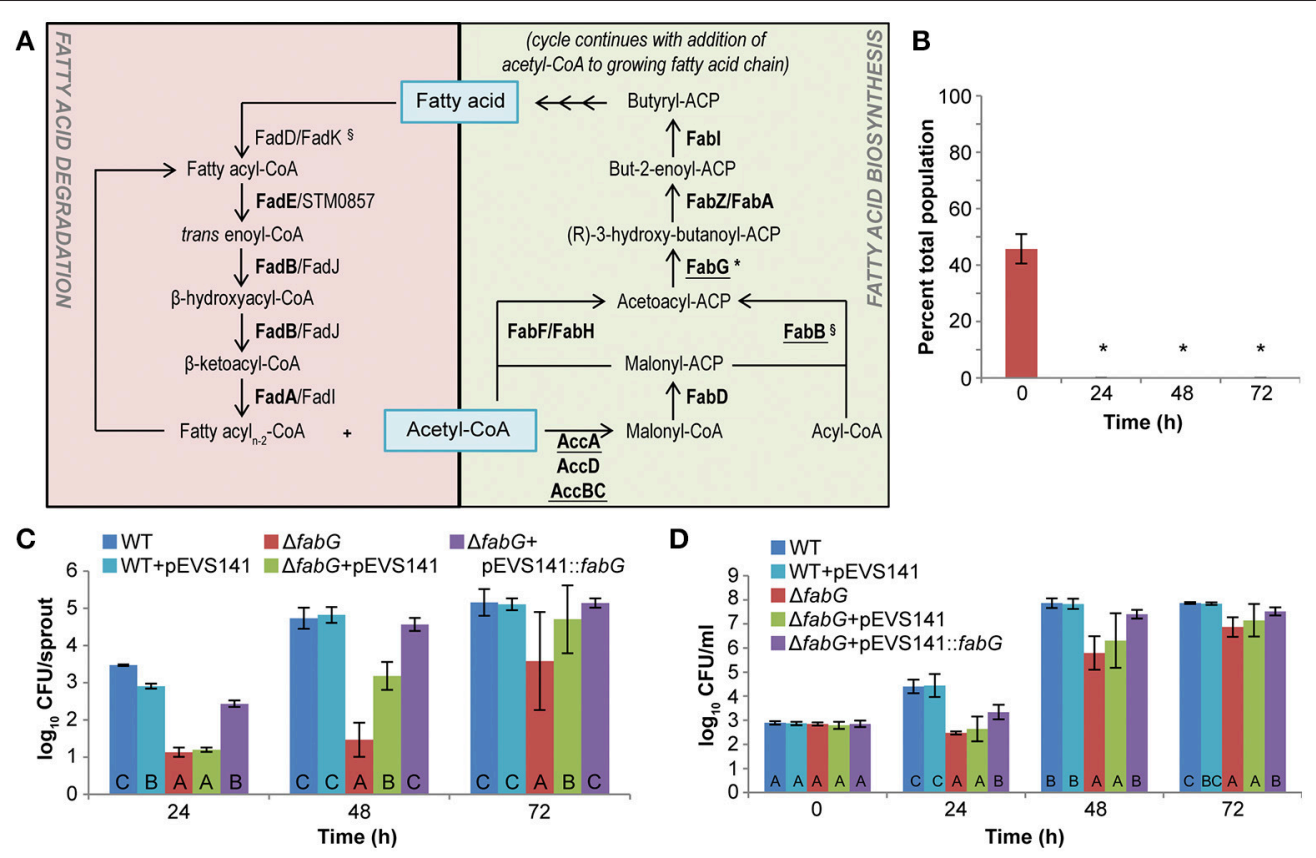

FIGURE 2 | The fatty acids metabolic network is required for S. enterica colonization of plants and animals. (A) Fatty acid degradation contributed to S. enterica colonization of animals (Steeb et al., 2013) whereas fatty acid biosynthesis was required for colonization of both animals and plants. Fatty acid network proteins were detected in proteomic screens of S. enterica during colonization of mice (bolded, Steeb et al., 2013) and growth in association with alfalfa seedlings (underlined, Kwan et al., 2015). § , significant colonization defect of mutant in mice (Barat et al., 2012; Steeb et al., 2013). *, significant colonization defect of mutant on alfalfa seedlings. (B) S. enterica SL1344 $\Delta$ fabG was non-competitive in alfalfa seedling colonization. Bars show the mean percent of the total population comprised of $\Delta$ fabG following a 1:1 co-inoculation with the isogenic WT. *, statistically different from $50 \%$ (Student's $t$-test, $n=3, p<0.05)$. (C,D) $\Delta$ fabG was defective in alfalfa seedling colonization (C) and growth in seedling exudates (D). These defects were complemented by addition of a plasmid-borne copy of fabG on pEVS141. Bars show the average population of $S$. enterica present on alfalfa sprouts (C) or the irrigation water containing seedling exudates (D). Letters indicate statistically different groups within each time point (Tukey's HSD, $n=45, p<0.05$ ). For all experiments, data shown are the means of 3 independent experiments with 3 replicates each. Error bars indicate the standard deviation.
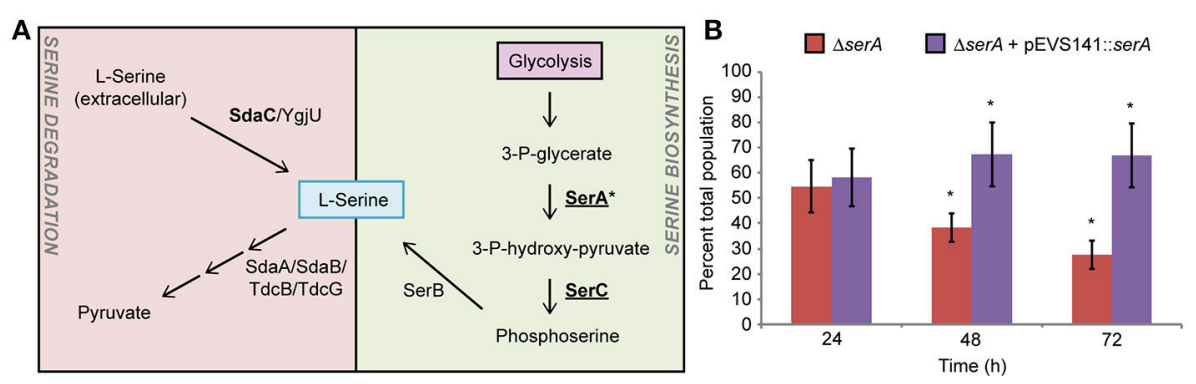

FIGURE 3 | Serine biosynthesis is required for S. enterica plant colonization. (A) Serine network proteins were detected in proteomic screens of S. enterica during colonization of mice (bolded, Steeb et al., 2013) and growth in association with alfalfa seedlings (underlined, Kwan et al., 2015). S. enterica SL1344 $\Delta$ serA was reduced in competitive fitness in alfalfa seedling colonization (B). Bars show the mean percent of the total population comprised of $\Delta$ serA following a $1: 1$ co-inoculation with the isogenic WT. *, statistically different from $50 \%$ (Student's $t$-test, $n=3, p<0.05$ ).

\section{manA Is Required for S. enterica Plant Colonization}

Proteomic data indicate that ManA is active during mouse spleen colonization and a manA mutant is less fit in infected mouse tissues (Steeb et al., 2013). A manA mutant was also non-competitive for alfalfa seedling colonization (Figure 1). ManA catalyzes the reversible isomerization of mannose-6phosphate into fructose-6-phosphate. This defect was partially complemented by a plasmid carrying a WT copy of manA (Figure 4A). The fitness of the manA mutant was fully restored by addition of $0.2 \mathrm{mM}$ mannose, but not $0.2 \mathrm{mM}$ fructose, suggesting that the directionality of the reaction catalyzed by manA in planta is toward production of mannose-6-phosphate (data not shown). Mannose-6-phosphate is a precursor for the biosynthesis of the surface polymers colanic acid and Oantigen. The competitive colonization defect of the manA 


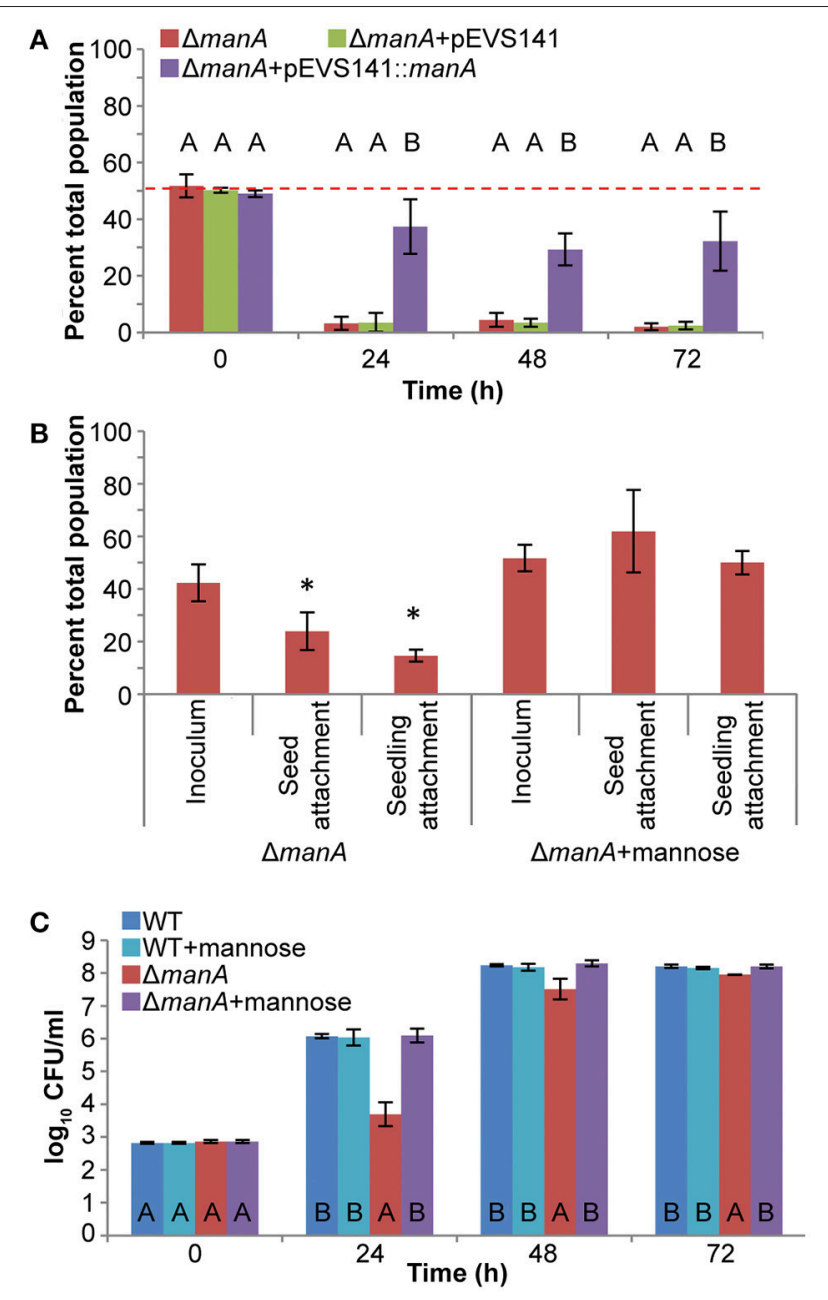

FIGURE 4 | Mannose is required for S. enterica plant colonization. (A) SL1344 $\triangle m a n A:: C m$ had a severe competitive colonization defect on alfalfa seedlings. Bars show the mean percent of the total population comprised of $\triangle$ manA following a 1:1 co-inoculation with the isogenic WT. * , statistically different from $50 \%$ (One-sample $t$-test, $n=3, p<0.05$ ). Letters above bars, statistically different groups within each time point (Tukey's HSD, $n=9, p<0.05$ ).

(B) Mannose contributed to $S$. enterica attachment to alfalfa seeds and 3 day-old seedlings. The attachment defect of $\triangle m a n A:: C m$ was fully complemented by addition of $0.2 \mathrm{mM}$ mannose. Bars show the mean percent of the total population comprised of $\triangle$ manA following a co-inoculation with the isogenic WT. *, statistically different from the inoculum (Paired $t$-test, $n=6, p$ $<0.05)$. (C) Mannose contributes to $S$. enterica growth in alfalfa seedling exudates in vivo. The growth defect of $\triangle$ manA::Cm was fully complemented by addition of $0.2 \mathrm{mM}$ mannose. Letters at the bottom of the bars, statistically different groups within each time point (Tukey's HSD, $n=36, p<0.05$ ). For all experiments, data shown are the means of 3 independent experiments with 3 replicates each. Error bars indicate the standard deviation.

mutant stemmed from defects in both attachment, to alfalfa seeds and seedlings, and growth (Figures 4B,C). These defects were fully complemented by the addition of $0.2 \mathrm{mM}$ mannose to the overnight culture (attachment) or the irrigation water (growth). The growth pattern of the manA mutant suggested that growth was delayed, but the growth rate was not impaired beyond the first $24 \mathrm{~h}$. During the first $24 \mathrm{~h}$, WT populations
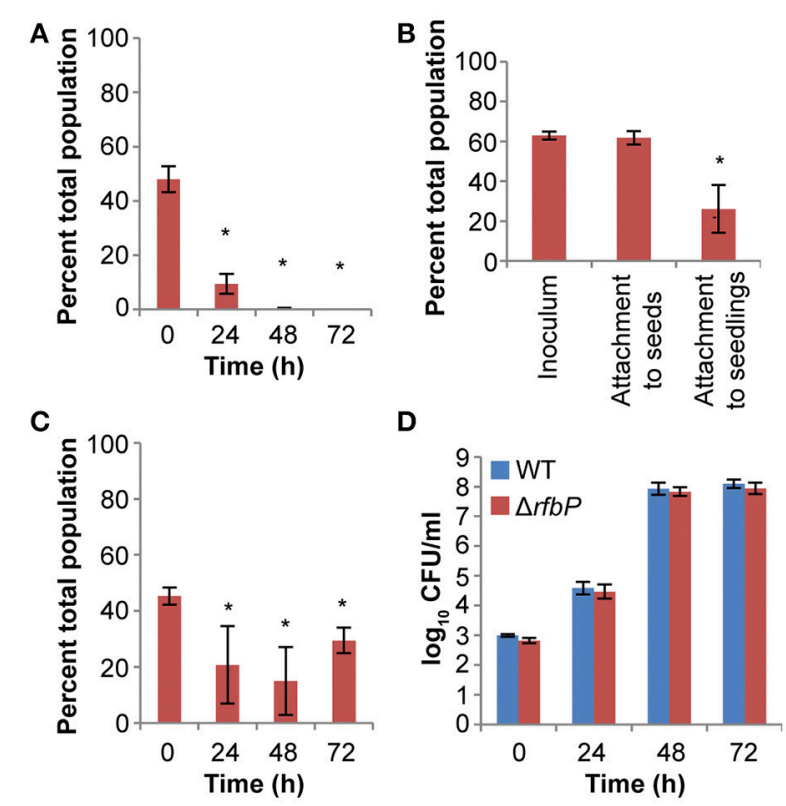

FIGURE 5 | O-antigen contributes to $S$. enterica plant colonization. (A) 14028S $\triangle r f b P$, an O-antigen mutant, was less competitive than the isogenic WT in alfalfa seedling colonization. * , statistically different from $50 \%$ (Student's $t$-test, $n=3, p<0.05$ ). (B) $O$-antigen contributed to attachment to 3 d-old seedlings but not seeds. *, statistically different from inoculum (Paired $t$-test, $n=6, p<0.05)$. (C,D) $\Delta r f b P$ had a subtle growth defect in alfalfa seedling exudates that is revealed only in competition with the isogenic WT. (C) *, statistically different from 50\% (Student's $t$-test, $n=3, p<0.05$ ). (D) *, statistically different from WT (Paired t-test, $n=6, p<0.05$ ). WT and mutant were inoculated at a 1:1 ratio for all competition experiments $(\mathbf{A}, \mathbf{B}, \mathbf{C})$. All data shown represent the means of 3 independent experiments with 3 replicates each. Error bars indicate the standard deviation.

increased $\sim 3 \log _{10}$ (1000-fold) whereas the manA mutant growth was severely restricted to $<1 \log _{10}(<10$-fold; Figure $4 \mathrm{C})$. The manA mutant resumed growth between $24-48 \mathrm{~h}$, increasing 3.8 $\log _{10}$, resembling WT replication during the $0-24 \mathrm{~h}$ period. Taken together, these data suggest that mannose may be a required metabolite that is absent from early seedling exudates. Alternately, manA may be required for plant host interactions.

\section{O-antigen Contributes to Distinct Steps in Plant Colonization}

We hypothesized that the colonization defects of the manA mutant were due to defects in the production of extracellular polysaccharides from the mannose metabolic pathway, either Oantigen or colanic acid. We tested this hypothesis by examining the competitive colonization of $g m d$ and $r f b P$ mutants against the WT. A gmd mutant, unable to produce the GDP-fucose, is defective in colanic acid biosynthesis and a $r f b P$ mutant fails to catalyze the first step in O-antigen assembly on the bacterial cell membrane. The gmd mutant was as competitive as the WT for seedling colonization and exhibited no attachment or growth defects (data not shown). The $r f b P$ mutant was less fit than the WT in a competitive colonization assay (Figure 5A). This colonization defect was due to deficiencies in attachment and 
competitive growth (Figures 5B,C, respectively). However, no growth defect was observed when the $r f b P$ mutant was singly inoculated into plant cultures (Figure 5D). Our data indicate that $\mathrm{O}$-antigen, but not colanic acid, contributed to distinct steps of S. enterica seedling colonization.

\section{DISCUSSION}

It is widely accepted that $S$. enterica adopts a cross-kingdom lifestyle, actively colonizing and persisting on plants in between infection of animal or human hosts (Barak and Schroeder, 2012). We hypothesized that this human pathogen would employ distinct metabolic strategies for colonization of crosskingdom hosts due to the inherent differences in plant and animal host physiology as well as the nature of the extracellular (plant) vs. intracellular (animal) niches. A recent mutant screen of metabolic mutant colonization of murine spleens gave us the opportunity to test our hypothesis. Direct comparison of the same set of $S$. enterica mutants in both environments is a powerful approach that avoids identification of artificial differences that may arise from comparisons based on disparate data sets. Whereas the SCV inside of animal cells contains high levels of reactive oxygen and nitrous oxide species and few sugars but sufficient amino acids, plant root exudates are aerobic and sugar-rich, but amino acid deficient. We were thus surprised to find that $S$. enterica generally depends on the same metabolic networks to exploit both plant and animal hosts.

Biosynthesis of essential cellular metabolites is a key metabolic function shared between the plant- and animal-associated lifestyles of $S$. enterica. However, the specific nutritional constraints on $S$. enterica growth differed between plant and animal hosts. Though amino acids were present in both host environments, as inferred from impaired colonization by mutants and measured depletion of these metabolites, amino acids were much more limiting during growth in plant seed exudates (alfalfa, lettuce, broccoli; Kwan et al., 2015 and this study) and tomato fruit (Han and Micallef, 2016) than in animal models of infection. Of the 13 amino acids which have been examined in murine models, only asparagine and proline biosynthesis were required for full fitness (Steeb et al., 2013; Jelsbak et al., 2014). In contrast, de novo biosynthesis of at least 8 different essential amino acids (out of 18 examined) were required in each plant environment for full $S$. enterica fitness (Kwan et al., 2015; de Moraes et al., 2017, and this study), though the exact amino acids varied by plant. Notably, methionine and tryptophan biosynthesis emerged as common requisites for the plant-associated lifestyle of $S$. enterica that are dispensable during animal infection. We recognize that levels of different amino acids will vary and depend on factors such as plant growth conditions, plant genotype, plant growth stage, as well as plant organ; yet, our study did examine root exudates from plants from three distinct orders while murine models are restricted to a single host (mouse) that carry a dysfunctional Slc1 1a1 allele which makes them highly susceptible to systemic Salmonella infections. Collectively, the data presented herein and in the current literature indicate that the host-associated lifestyles of
S. enterica, whether on plants or animals, involve exploitation of host nutrients as well as de novo biosynthesis of metabolites insufficiently available from the host.

The much greater variety of amino acids that must by produced endogenously during plant-associated growth compared to animal niches reflects the general low availability of these nutrients from plant hosts as well as the high bacterial titers $\left(\geq 10^{7} \mathrm{CFU} / \mathrm{ml}\right)$ in plant environments. For example, the average concentration of every amino acid except threonine in alfalfa seedling exudates was less than $70 \mathrm{uM}$, which is 3-185 times lower than reported in murine spleen tissue (Xiao et al., 2016). Plants may tightly control production and release of certain nutrients, e.g., cysteine, due to their toxicity (Figure S1). Thus, even dedicated plant colonists such as $P$. fluorescens and $P$. tolaasii must synthesize these metabolites to succeed in the plant environment (Simons et al., 1997; Chung et al., 2014). Because amino acid restriction is common in the plant environment, amino acid prototrophy appears as a universal plant colonization trait for a variety of plant-associated bacteria.

Utilization of multiple, diverse nutrients appears to be a general metabolic colonization strategy of S. enterica. During typhoid fever in mice, spleen colonization by $S$. enterica depended on the simultaneous exploitation of seven host nutrients - glycerol, fatty acids, $\mathrm{N}$-acetylglucosamine, gluconate, glucose, lactate, and arginine (Steeb et al., 2013). During alfalfa seedling colonization, S. enterica was inferred to use glycerol, glucose, galactose, ribose, maltose, nucleosides, arginine, and proline to support its growth (this study), as mutants in these metabolic pathways were less fit. The exploitation of a greater range of sugars in the plant environment reflects the richness of sugars in plant exudates. In both plant and animal hosts, glycerol and its derivative glycerol-3-phosphate were metabolic drivers of $S$. enterica colonization. Mutants impaired in the transport and/or catabolism of these compounds were up to 10-fold less fit than the WT in competitive colonization of both hosts. Because these mutants were not non-competitive, we conclude that glycerol and glycerol-3-phosphate are not essential metabolic determinants of host colonization by $S$. enterica. Overall, most individual nutrients appeared to have small contributions to bacterial colonization. The availability or use of specific nutrients did not determine plant colonization ability; rather, high levels of S. enterica colonization is supported by the contributions of many plant-derived nutrients. This diversified metabolic strategy may enable S. enterica to colonize diverse hosts and better adapt to changes in the host environment, such as the alteration of plant exudate composition during plant development. However, the robust metabolism of S. enterica and the rich and dynamic nature of plant exudates present a complex challenge for designing strategies aimed at restricting or reducing enteric human pathogen growth on food crops. Identifying essential nutrients that S. enterica either cannot biosynthesize, e.g., minerals, or cannot import will be key to this endeavor.

While there are many similarities in S. enterica metabolism during plant and animal colonization, we highlight in this work three distinct differences in the metabolic networks used to support each bacterial lifestyle. For example, host differences and metabolic preferences lead to differential usage of the 
metabolic network surrounding fatty acids. Proteomic data indicate that this metabolic network is active during mouse spleen colonization and alfalfa seedling-associated growth (Steeb et al., 2013; Kwan et al., 2015). Fatty acid biosynthesis by $S$. enterica is likely important for repair of cell membranes damaged during infection and production of new membrane components for replicating cells. Disruption of fatty acid biosynthesis in a $\mathrm{fabB}$ mutant resulted in a 100,000-fold loss in competitive fitness $\left(\log _{2}\right.$ (competitive index $)<-16.7$ ) and rapid clearance of the bacterium from infected mice (Becker et al., 2006; Barat et al., 2012). The failure of a $f a b G$ fatty acid biosynthetic mutant to grow during the first $24 \mathrm{~h}$ in alfalfa seedling exudates and the restoration of its growth ability thereafter suggests that fatty acids were not available to $S$. enterica in early seedling exudates but became available as the seedlings matured. Even as this nutrient became available, it was not a preferred nutrient source for $S$. enterica based on the WT phenotype of the fadD fadK fatty acid utilization mutant. This result is consistent with the use of sugars as preferred carbon sources, which are abundant in plant exudates. This conclusion contrasts with $S$. enterica nutrition during systemic murine infection where fatty acids were a major nutrient source (Steeb et al., 2013). Though fatty acid degradation was not necessary for the phases of plant-associated growth (lag to late log phases) examined in this study, partly due to the lack of this nutrient in very early seedling exudates, this metabolic function may become more important as bacterial cells enter the less biosynthetically-active stationary phase. At this point, maintenance and repair of cell membranes will likely shift from use of phospholipids synthesized de novo to modification or degradation and repurposing of existing cellular lipids. A recent study of stationary phase S. enterica in tomato fruits appears to support this conclusion (de Moraes et al., 2017). Fatty acid degradation may be an example of divergent use of a single metabolic network, for cellular maintenance vs. nutrition, during plant and animal colonization.

Serine metabolism is another example of a distinct difference between S. enterica plant and animal colonization. SerA and SerC, two enzymes involved in serine biosynthesis via the glycolysis intermediate 3-phosphoglycerate, are produced both during growth in germinating alfalfa exudates (Kwan et al., 2015) and during animal colonization (Becker et al., 2006; Steeb et al., 2013). In contrast, the serine transporter SdaC and serine deaminases SdaA and SdaB were detected only during animal infection. Mutant analysis indicates that serine biosynthesis is required for alfalfa root colonization (this study) but dispensable during murine infection (Jelsbak et al., 2014). Serine biosynthesis occurs during both plant and animal colonization but is only essential for root colonization suggesting that sufficient serine may be available in animals or serine synthesis from glycine is energetically favorable during animal colonization. The latter is supported by the finding that the $g v c$ operon, involved in this glycine-to-serine conversion, is active during animal infection (Eriksson et al., 2003). Taken together, the data suggests that serine is limited in both murine spleens and alfalfa root exudates but $S$. enterica uses different biosynthetic pathways to overcome that deficit during its plant- and animal-associated lifestyles.
We also examined the metabolic network surrounding mannose. A manA mutant was severely compromised in competitive colonization of both plants and animals. ManA, or phosphomannose isomerase (Pmi), catalyzes the reversible isomerization of mannose-6-phosphate into fructose-6phosphate, a glycolytic intermediate. In both plants and animals, the reaction catalyzed by ManA is believed to operate in the reverse direction, diverting fructose-6-phosphate from glycolysis to yield mannose-6-phosphate (Steeb et al., 2013, this study). Mannose-6-phosphate is a precursor for the nucleotide sugars that form the backbones of the common enterobacterial extracellular polysaccharides colanic acid and O-antigen. A pmi mutant is avirulent in mice, and the loss of competitive colonization fitness in a manA mutant is attributed to loss of $\mathrm{O}$ antigen (Collins et al., 1991; Steeb et al., 2013). O-antigen delays immune recognition and confers resistance to complementmediated killing and antimicrobial peptides in animal models of infection (Duerr et al., 2009; Ilg et al., 2009). However, we found no role for manA in resistance to ROS, acidity, flavone, or the antimicrobial peptide polymyxin B in plant exudates (data not shown). In fact, O-antigen deficiency only partially explained the phenotype of the manA mutant in planta. Oantigen contributed to seedling but not seed attachment, and competitive growth fitness in the plant environment but not the inherent ability to grow in the absence of competition. We also found no role for colanic acid in S. enterica seed or seedling attachment, colonization, or plant-associated growth. These results are consistent with a previous report that identified a role for $\mathrm{O}$-antigen, but not colanic acid, in alfalfa sprout attachment and colonization by $S$. enterica sv. Enteriditis (Barak et al., 2007). However, colanic acid has been reported to contribute to desiccation tolerance on leaf surfaces (Cowles et al., 2016). The results from this study suggest that physiological functions in addition to $\mathrm{O}$-antigen and colanic acid are impaired in a manA mutant, which is also reduced in seed attachment and severely compromised in the ability to grow during the first $24 \mathrm{~h}$ following inoculation into a germinating seedling culture. We hypothesize that mannose-6-phosphate and/or a downstream metabolite essential for plant-associated growth is absent from early seed exudates and must be biosynthesized endogenously by S. enterica to permit bacterial proliferation.

Taken together, this study shows that there is high overlap in the metabolic networks that are required for both the plantand animal-associated lifestyles of S. enterica. However, some of these networks can be used differently to enable $S$. enterica to colonize each host, reflecting the chemical environment provided by the host. Common, simple molecules such as sugars, organic acids, and amino acids comprise the diet of S. enterica in both plants and animals. This finding was initially surprising to us because plants and animals are physiologically distinct and we expected unique $S$. enterica metabolic profiles in these environments. However, plants and animals share the same basic cellular metabolites (i.e., glucose, glycerol, amino acids, etc.) and pathways; it is understandable that similar host nutrients support $S$. enterica colonization of plants and the SCV inside of animal cells. Thus, the success of $S$. enterica in both host environments is partly due to similar available metabolites from plant and animal 
hosts. Additionally, the metabolic capacity of the bacteriumcharacterized by nutritional versatility, biosynthesis of limiting metabolites, and co-opting virulence pathways (e.g., the mannose metabolic network) for plant colonization-likely contribute to the ability of $S$. enterica to exploit cross-kingdom hosts.

\section{CONCLUSIONS}

We have provided fundamental information about how a human enteric pathogen grows on and colonizes plants. This advancement is an important step to understand the biology of S. enterica, the leading bacterial pathogen of food-borne illness which is now most commonly linked to consumption of fresh produce. We examined S. enterica mutants defective in the metabolism of diverse nutrients for their ability to colonize plants in order to identify the traits that contribute to its success. A comparison with the metabolic requirements for splenic colonization revealed few differences in metabolic network use, e.g., amino acid and fatty acid metabolism and exopolysaccharide production. However, pathways within these networks served different functions facilitating S. enterica colonization of diverse niches. To thrive in distinct niches that span multiple kingdoms of life, S. enterica exhibits metabolic versatility and adaptation of physiological pathways for alternate functions. Having the capacity to colonize the food source (plants) of a preferred host (omnivore or herbivore) allows fluidity in the lifecycle of S. enterica.

\section{REFERENCES}

Barak, J. D., and Schroeder, B. K. (2012). Interrelationships of food safety and plant pathology: the life cycle of human pathogens on plants. Annu. Rev. Phytopathol. 50, 241-266. doi: 10.1146/annurev-phyto-081211-172936

Barak, J. D., Jahn, C. E., Gibson, D. L., and Charkowski, A. O. (2007). The role of cellulose and O-antigen capsule in the colonization of plants by Salmonella enterica. Mol. Plant Microbe Interact. 20, 1083-1091. doi: 10.1094/MPMI-20-9-1083

Barat, S., Steeb, B., Mazé, A., and Bumann, D. (2012). Extensive in vivo resilience of persistent Salmonella. PLoS ONE 7:e42007. doi: 10.1371/journal.pone.0042007

Becker, D., Selbach, M., Rollenhagen, C., Ballmaier, M., Meyer, T. F., Mann, M., et al. (2006). Robust Salmonella metabolism limits possibilities for new antimicrobials. Nature 440, 303-307. doi: 10.1038/nature04616

Bishop, A. L., and Davis, R. M. (1990). Internal decay of onions caused by Enterobacter cloacae. Plant Dis. 74, 692-694. doi: 10.1094/PD-74-0692

Bruton, B. D., Mitchell, F., Fletcher, J., Pair, S. D., Wayadande, A., Melcher, U., et al. (2003). Serratia marcescens, a phloem-colonizing, squash bug -transmitted bacterium: causal agent of cucurbit yellow vine disease. Plant Dis. 87, 937-944. doi: 10.1094/PDIS.2003.87.8.937

Callejón, R. M., Rodríguez-Naranjo, M. I., Ubeda, C., Hornedo-Ortega, R., GarciaParrilla, M. C., and Troncoso, A. M. (2015). Reported foodborne outbreaks due to fresh produce in the United States and European Union: trends and causes. Foodborne Pathog. Dis. 12, 32-38. doi: 10.1089/fpd.2014.1821

Chatterjee, S., Almeida, R. P., and Lindow, S. (2008). Living in two worlds: the plant and insect lifestyles of Xylella fastidiosa. Annu. Rev. Phytopathol. 46, 243-271. doi: 10.1146/annurev.phyto.45.062806.094342

Chung, I. Y., Kim, Y. K., and Cho, Y. H. (2014). Common virulence factors for Pseudomonas tolaasii pathogenesis in Agaricus and Arabidopsis. Res. Microbiol. 165, 102-109. doi: 10.1016/j.resmic.2013.12.001

Collins, L. V., Attridge, S., and Hackett, J. (1991). Mutations at rfc or pmi attenuate Salmonella typhimurium virulence for mice. Infect. Immun. 59, 1079-1085.

\section{AUTHOR CONTRIBUTIONS}

GK and JB conceived the study and wrote the manuscript. GK, JB, $\mathrm{KC}$, TP, and DA-N analyzed the data. GK conducted the majority of the assays. TP conducted the metabolomics assays. BP and KC participated in mutant phenotyping. All authors have read and approved the final manuscript.

\section{FUNDING}

Funding was provided by USDA-HATCH grant no. WIS01696 and the Food Research Institute at UW-Madison. The funders had no role in study design, data collection and interpretation, decision to publish, or preparation of this work for publication.

\section{ACKNOWLEDGMENTS}

We thank Dirk Bumann for providing a library of S. enterica SL1344 metabolic mutants, Christopher Waters for pEVS141, and Louise Pitcher and Tyler Engel for technical assistance.

\section{SUPPLEMENTARY MATERIAL}

The Supplementary Material for this article can be found online at: https://www.frontiersin.org/articles/10.3389/fmicb. 2018.00695/full\#supplementary-material

Conover, W. J. (2012). The rank transformation-an easy and intuitive way to connect many non-parametric methods to their parametric counterparts for seamless teaching introductory statistics courses. Wiley Interdiscipl. Rev. 4, 432-438. doi: 10.1002/wics.1216

Cowles, K. N., Willis, D. K., Engel, T. N., Jones, J. B., and Barak, J. D. (2016). Diguanylate cyclases adra and stm1987 regulate Salmonella enterica exopolysaccharide production during plant colonization in an environment-dependent manner. Appl. Environ. Microbiol. 82, 1237-1248. doi: 10.1128/AEM.03475-15

Datsenko, K. A., and Wanner, B. L. (2000). One-step inactivation of chromosomal genes in Escherichia coli K-12 using PCR products. Proc. Natl. Acad. Sci. U.S.A. 97, 6640-6645. doi: 10.1073/pnas.120163297

de Moraes, M. H., Desai, P., Porwollik, S., Canals, R., Perez, D. R., Chu, W., et al. (2017). Salmonella persistence in tomatoes requires a distinct set of metabolic functions identified by transposon insertion sequencing. Appl. Environ. Microbiol. 83:e03028-16. doi: 10.1128/AEM.03028-16

Duerr, C. U., Zenk, S. F., Chassin, C., Pott, J., Gütle, D., Hensel, M., et al. (2009). $\mathrm{O}$-antigen delays lipopolysaccharide recognition and impairs antibacterial host defense in murine intestinal epithelial cells. PLoS Pathog. 5:e1000567. doi: 10.1371/journal.ppat.1000567

Dunn, A. K., Millikan, D. S., Adin, D. M., Bose, J. L., and Stabb, E. V. (2006). New rfp- and pES213-derived tools for analyzing symbiotic vibrio fischeri reveal patterns of infection and lux expression in situ. Appl. Environ. Microbiol. 72, 802-810. doi: 10.1128/AEM.72.1.802-810.2006

El-Hamalawi, Z. A., and Erwin, D. C. (1986). Components in alfalfa root extract and root exudate that increase oospore germination of Phytophthora megasperma f. sp. medicaginis. Phytopath 76, 508-513. doi: 10.1094/Phyto-76-508

Eriksson, S., Lucchini, S., Thompson, A., Rhen, M., and Hinton, J. C. (2003). Unravelling the biology of macrophage infection by gene expression profiling of intracellular Salmonella enterica. Mol. Microbiol. 47, 103-118. doi: 10.1046/j.1365-2958.2003.03313.x 
Gutnick, D., Calvo, J. M., Klopotowski, T., and Ames, B. N. (1969). Compounds which serve as the sole source of carbon or nitrogen for Salmonella typhimurium LT-2. J. Bacteriol. 100, 215-219.

Han, S., and Micallef, S. A. (2016). Environmental metabolomics of the tomato plant surface provides insights on Salmonella enterica colonization. Appl. Environ. Microbiol. 82, 3131-3142. doi: 10.1128/AEM.00435-16

Hao, L.-Y., Willis, D. K., Andrews-Polymenis, H., McClelland, M., and Barak, J. D. (2012). Requirement of siderophore biosynthesis for plant colonization by Salmonella enterica. Appl. Environ. Microbiol. 78, 4561-4570. doi: 10.1128/AEM.07867-11

Harvey, P. C., Watson, M., Hulme, S., Jones, M. A., Lovell, M., Berchieri, A., et al. (2011). Salmonella enterica serovar typhimurium colonizing the lumen of the chicken intestine grows slowly and upregulates a unique set of virulence and metabolism genes. Infect. Immun. 79, 4105-4121. doi: 10.1128/IAI.01390-10

Hejazi, A., and Falkiner, F. R. (1997). Serratia marcescens. J. Med. Microbiol. 46, 903-912. doi: 10.1099/00222615-46-11-903

Hoiseth, S. K., and Stocker, B. A. (1981). Aromatic-dependent Salmonella typhimurium are non-virulent and effective as live vaccines. Nature 291, 238-239. doi: 10.1038/291238a0

Ilg, K., Endt, K., Misselwitz, B., Stecher, B., Aebi, M. , and Hardt,W. D. (2009). $\mathrm{O}$-antigen-negative Salmonella enterica serovar typhimurium is attenuated in intestinal colonization but elicits colitis in streptomycin-treated mice. Infect. Immun. 77, 2568-2575. doi: 10.1128/IAI.01537-08

Jelsbak, L., Hartman, H., Schroll, C., Rosenkrantz, J. T., Lemire, S., Wallrodt, I., et al. (2014). Identification of metabolic pathways essential for fitness of Salmonella typhimurium in vivo. PLoS ONE 9:e101869. doi: 10.1371/journal.pone.0101869

Kirzinger, M. W., Nadarasah, G., and Stavrinides, J. (2011). Insights into cross-kingdom plant pathogenic bacteria. Genes 2, 980-997. doi: $10.3390 /$ genes 2040980

Kwan, G., Pisithkul, T., Amador-Noguez, D., and Barak, J. (2015). De novo amino acid biosynthesis contributes to Salmonella enterica growth in alfalfa seedling exudates. Appl. Environ. Microbiol. 81, 861-873. doi: 10.1128/AEM.02985-14

Mercier, J., and Lindow, S. E. (2000). Role of leaf surface sugars in colonization of plants by bacterial epiphytes. Appl. Environ. Microbiol. 66, 369-374. doi: 10.1128/AEM.66.1.369-374.2000

Miller, W. G., Leveau, J. H., and Lindow, S. E. (2000). Improved gfp and inaZ broad-host-range promoter-probe vectors. Mol. Plant Microbe Interact. 13, 1243-1250. doi: 10.1094/MPMI.2000.13.11.1243

Nelson, E. B. (2004). Microbial dynamics and interactions in the spermosphere. Annu. Rev. Phytopathol. 42, 271-309. doi: 10.1146/annurev.phyto.42.121603.131041
Painter, J. A., Hoekstra, R. M., Ayers, T., Tauxe, R. V., Braden, C. R., Angulo, F. J., et al. (2013). Attribution of foodborne illnesses, hospitalizations, and deaths to food commodities by using outbreak data, united states, 1998-2008. Emerging Infect. Dis. 19, 407-415. doi: 10.3201/eid1903. 111866

Roberts, D. P., Baker, C. J., McKenna, L., Liu, S., Buyer, J. S., and Kobayashi, D., Y. (2009). Influence of host seed on metabolic activity of Enterobacter cloacae in the spermosphere. Soil Biol. Biochem. 41, 754-761. doi: 10.1016/j.soilbio.2009.01.010

Sanders, W. E. Jr. and Sanders, C. C. (1997). Enterobacter spp.: pathogens poised to flourish at the turn of the century. Clin. Microbiol. Rev. 10, 220-241.

Santiviago, C. A., Reynolds, M. M., Porwollik, S. S., Choi, H., Long, F., AndrewsPolymenis, H. L., et al. (2009). Analysis of pools of targeted Salmonella deletion mutants identifies novel genes affecting fitness during competitive infection in mice. PLoS Pathog. 5:e1000477. doi: 10.1371/journal.ppat. 1000477

Simons, M., Permentier, H. P., de Weger, L. A., Wijffelman, C. A., and Lugtenberg, B. J. J. (1997). Amino acid synthesis is necessary for tomato root colonization by pseudomonas fluorescens strain wcs365. Mol. Plant-Microbe Interact. 10, 102-106. doi: 10.1094/MPMI.1997.10.1.102

Steeb, B., Claudi, B., Burton, N. A., Tienz, P., Schmidt, A., Farhan, H., et al. (2013). Parallel exploitation of diverse host nutrients enhances Salmonella virulence. PLoS Pathog. 9:e1003301. doi: 10.1371/journal.ppat. 1003301

Wiedemann, A., Virlogeux-Payant, I. A., Chaussé, M., Schikora, A., and Velge, P. (2014). Interactions of Salmonella with animals and plants. Front. Microbiol. 5:791. doi: 10.3389/fmicb.2014.00791

Xiao, X., Hu, M., Liu, M., and Hu, J. (2016). 1H NMR metabolomics study of spleen from C57BL/6 mice exposed to gamma radiation. Metabolomics 6:165. doi: 10.4172/2153-0769.1000165

Conflict of Interest Statement: The authors declare that the research was conducted in the absence of any commercial or financial relationships that could be construed as a potential conflict of interest.

Copyright (c) 2018 Kwan, Plagenz, Cowles, Pisithkul, Amador-Noguez and Barak. This is an open-access article distributed under the terms of the Creative Commons Attribution License (CC BY). The use, distribution or reproduction in other forums is permitted, provided the original author(s) and the copyright owner are credited and that the original publication in this journal is cited, in accordance with accepted academic practice. No use, distribution or reproduction is permitted which does not comply with these terms. 